\title{
Relevance of the T cell Receptor-Ligand Avidity for Immunity to Infection
}

\author{
Magdalena Nauerth1, Kristof Wing ${ }^{1,2}$, Heinrich Körner ${ }^{2}$ and Dirk H Busch ${ }^{1,3,4^{*}}$ \\ 1 Institute for Medical Microbiology, Immunology and Hygiene, Technische Universität München, Munich, Germany \\ ${ }^{2}$ Menzies Institute for Medical Research, Medical Science Precinct, Hobart, Tasmania, Australia \\ ${ }^{3}$ DZIF - National Centre for Infection Research, Munich, Germany \\ ${ }^{4}$ Focus Group "Clinical Cell Processing and Purification”, Institute for Advanced Study, Technische Universität München, Munich, Germany
}

\begin{abstract}
Cytotoxic lymphocytes are critical for fighting viral and certain bacterial infections. Therefore, assessing the quality of cytotoxic T cell responses might have important clinical implications. TCR-pMHC binding (avidity) is a key determinant of T cell quality. Here we review currently available technologies for the measurement of TCR-pMHC avidity and their potential relevance for translational applications.
\end{abstract}

Keywords: T cell; T cell receptor; Avidity; Intracellular infections; Immunotherapies

\section{Introduction}

Chronic infections with viruses and intracellular bacteria remain a leading cause of morbidity and mortality worldwide. The Human Immunodeficiency Viruses (HIV) account for over $4 \%$ of the Years of Life Lost globally [1], continue to be largely incurable, and have increasing rates of therapeutic resistance [2]. Mycobacterium tuberculosis is estimated to latently infect between two and three billion people worldwide [3], with mortality in active, extensively drug resistant (XDR-TB) cases approaching $50 \%$ in spite of rigorous treatment [4]. Similarly, cytomegalovirus (CMV) and Epstein-Barr virus (EBV) cause significant morbidity and morality in immunosuppressed patients, and are regularly refractory to current therapies [5]. The treatment of each of these conditions is associated with, at times, severe toxicities that limit management and prevent curative treatment [6-8]. These inadequacies have led to the investigation of novel immunotherapies, including vaccination and adoptive cell therapy (ACT).

The control and clearance of intracellular pathogens usually requires robust cytolytic immunity, pivotally mediated by antigenspecific $\mathrm{CD}^{+} \mathrm{T}$ cells [9]. Highly diverse repertoires of $\mathrm{CD} 8^{+} \mathrm{T}$ cells in the pool of lymphocytes, with each cell expressing a unique $\mathrm{T}$ cell receptor (TCR) on its surface, are provided by thymic development and selection. These TCRs bind, together with the CD8 co-receptor, to major histocompatibility complex I molecules loaded with cognate peptides (pMHC), which are expressed on the surface of target cells. Infected cells presenting peptides from the intracellular pathogen can thereby be specifically recognised by $\mathrm{T}$ cells, and are killed or modulated due to the consequent effector functions of the T cell. In this context, the term 'T cell avidity' describes the efficiency of these effector functions after antigen encounter and is principally affected by the binding strength between the TCR, its cognate pMHC ligand, and associated co-receptors, the so called 'structural avidity'. The structural avidity of TCR-pMHC binding can be expressed by the equilibrium constant $K_{D}$, a ratio between the association $\left(k_{o n}\right)$ rate and the dissociation $\left(k_{o f f}\right)$ rate $[10,11]$ with low equilibrium constants indicative of high avidity TCR-pMHC complexes. Interactions characterised by high structural avidities are correlated with increased $\mathrm{T}$ cell functional avidities $[10,12]$, with a major determinant of the structural TCR avidity being the $k_{\text {off }}$-rate $[10,13]$. There is growing interest in identifying $\mathrm{T}$ cells with high avidity TCRs, as they have been shown to possess superior efficacy towards target cells in vivo and in vitro [14-16]. Given that high avidity $\mathrm{T}$ cells recognize targets earlier and mediate superior effector functions [14], their generation and precise detection becomes an important aspect for the development of more effective anti-infective immunotherapies.

\section{Measuring TCR-pMHC Interaction}

Functional assays, such as interferon- $\gamma$ synthesis, interleukin-2 release or in vitro cell-mediated lysis, can be strongly affected by the expression level of TCRs and co-receptors, and alterations in the signaling cascade [17], and are cumbersome to undertake. Several different methods have been developed, which aim to assess the structural avidity of TCR-pMHC binding in order to overcome the limitations of functional assays, as proxies for functionality. Surface Plasmon Resonance (SPR) (Figure 1a), initially developed by Liedberg et al. [18], has been adapted to assess the binding of TCRs or MHCs devoid of co-receptors, with one member of the pair being soluble and one substrate-bound, by detecting changes in the mass of the bound complexes. Typically, this technique is complicated by the technically challenging and labour intensive production of high-purity soluble TCRs and MHC molecules. Furthermore, the contribution of the CD8 co-receptor and other potentially involved surface molecules to binding is unable to be interrogated, thus preventing assessment of the physiological conditions present at the T cell surface.

Conversely, assays based on multimer binding (Figure 1b) and dissociation (Figure 1c) attempt to measure the structural avidity of TCR-pMHC binding on the surface of living T cells $[13,19]$. These assays monitor the dissociation of multimeric complexes, can thus have half-lives of dissociation in the range of hours to days, are strongly influenced by the quality of multimer reagents, as well as the concentration and affinity of MHC-blocking antibodies used, a

*Corresponding author: Dirk H Busch, Institute for Medical Microbiology, Immunology and Hygiene, Technische Universität München, Munich, Germany, Tel: +49 89 28901; E-mail: dirk.busch@tum.de

Received February 29, 2016; Accepted March 24, 2016; Published March 31 2016

Citation: Nauerth M, Wing K, Körner H, Busch DH (2016) Relevance of the T cell Receptor-Ligand Avidity for Immunity to Infection. J Microb Biochem Technol 8 : 131-135. doi: 10.4172/1948-5948.1000275

Copyright: @ 2016 Nauerth M, et al. This is an open-access article distributed under the terms of the Creative Commons Attribution License, which permits unrestricted use, distribution, and reproduction in any medium, provided the original author and source are credited. 

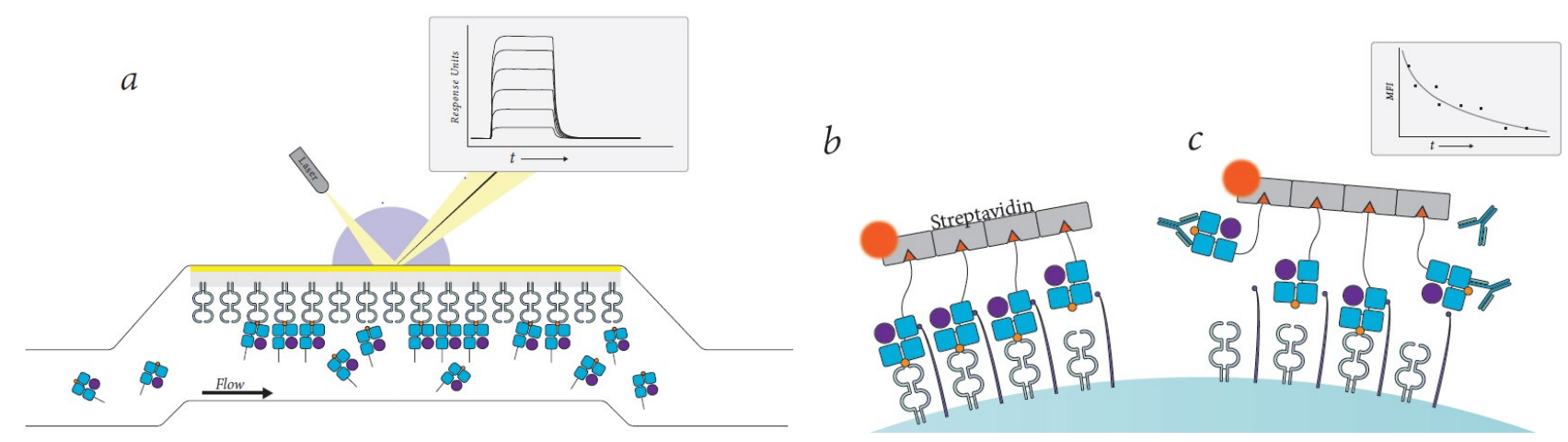

Figure 1: Methodologies for the quantification of TCR Avidity

a. Surface Plasmon Resonance: This strategy detects changes in the optical qualities of a gold-coated biosensor, which reacts when minute forces are applied to it. Underlying this surface is an organic matrix which binds macromolecules such as TCRs, immobilising them on the lumenal surface of a flow cell. Passing pMHC molecules over the lumenal surface facilitates the interaction and binding of TCR-pMHC complexes, resulting in an increase in mass bound to the organic matrix, an increase in force applied to the sensor chip, and thus alteration of the surface plasmon resonance of the gold surface. Subsequent dissociation of the TCR-pMHC complexes results in the system returning to baseline, permitting assessment the kon and koff -rates.

b. MHC multimer binding: Recombinant pMHC molecules are multimerised to a fluorescently labelled streptavidin molecule via C-terminal biotin conjugates generating a stable multimer, binding stably to surface expressed TCRs. Dissociation events are individually unable to abrogate binding of the multimer as they are compensated for by the repetitive re-binding of neighbouring MHCs, resulting in stable fluorescent identification of cells.

c. Multimer dissociation assay: Addition of reagents, such as anti-MHC-I antibodies prevents stochastic re-association events and the maintenance of equilibrium, eventually resulting in dissociation of the multimeric complex. Analysis of fluorescent intensity at pre-determined time points each typically some hours apart, enables the approximation of a fluorescent decay curve, a derivative of the koff -rate of an interaction.

pre-requisite for the assay as it permits dissociation of the multimeric complex [20]. These factors result in significant inter-assay and interinvestigator variability, negatively effecting the reliability of this assay.

In an effort to overcome these inadequacies, Huang et al. [21] and Huppa et al. [22] have developed two-dimensional TCR-pMHC binding assays to better approximate the physiological situation, where both binding partners are embedded in 'two-dimensional' membranes. These assays provide valuable insights into TCR-pMHC physiology, but have thus far been unable to lend themselves to high-throughput translational applications.

\section{Determining T cell Avidity: The TCR-Ligand $k_{\text {off }}$-Rate Assay}

To enable the rapid and accurate measurement of truly monomeric TCR-pMHC interactions on living T cells, we developed the TCRLigand $k_{\text {off }}$-rate assay (Figure 2) based on reversible MHC multimers, so-called MHC StrepTamers [23]. Fluorescently-labelled MHC-I molecules, which associate with their cognate TCRs are multimerized onto StrepTactin molecules via C-terminal StrepTag sequences, permitting the stable identification of living $\mathrm{CD}^{+} \mathrm{T}$ cells via cooperative binding (Figure 2a). In the presence of D-biotin, the StrepTag is very rapidly displaced from the Strep Tactin molecule, causing disintegration of the multimer complex, whilst leaving monomeric MHC-I molecules bound to their cognate TCRs (Figure 2b). Dissociation of these MHC molecules from the surface of the $\mathrm{T}$ cell is visible as a decay in fluorescent intensity over time, readily permitting the assessment of the $k_{\text {off }}$-rate (Figure 2c) [24].

We analysed human $\mathrm{T}$ cells specific for CMV from healthy donors' PBMCs via the fluorescence microscopy $k_{\text {off }}$-rate assay, and demonstrated high reproducibility between experiments, dissociations, and even unknown identical clones [24]. Correlation of the $k_{\text {off }}$ rate and ex vivo cytokine and cytolytic assays demonstrated a clear association between the $k_{\text {off }}$-rate and functionality. Specifically, we have not yet found any endogenous epitope-specific $\mathrm{T}$ cell which exhibits fast $k_{o f f}$-rates and still high functionality [17], a constellation that could be achieved by a predominantly fast $k_{o n}-$ rate. For CMVspecific CD8 ${ }^{+} \mathrm{T}$ cells, differences in $k_{\text {off }}$-rate again correlated with the functional competence of the respective populations. Furthermore, we have demonstrated a clear correlation between protectivity and the $k_{\text {off }}$ -rate of T cells used in adoptive T cell transfer, in preclinical murine models of Listeria moncytogenes and murine CMV infection. In the meantime, others have also developed TCR-pMHC $k_{\text {off }}$-rate assays based on the same principles as our Streptamer based assay [25]. Their methodologies have found a clear correlation between the TCR-pMHC $k_{o f f}$-rate and the functionality of the analysed cells [26], supporting our previous findings.

Altogether, our data and the published work of other groups indicates that the $k_{\text {off }}$-rate is an important parameter for the identification and selection of highly functional $\mathrm{T}$ cells for different therapeutic and diagnostic applications [17,27].

\section{Applications of the TCR-Ligand $\boldsymbol{k}_{\text {off }}$-Rate Assay}

Adoptive $\mathrm{T}$ cell transfer experiments in preclinical mouse models have clearly demonstrated the superior protective capacity of $\mathrm{T}$ cells with long TCR-pMHC dissociation half-lives and thus low $k_{\text {off }}$-rates [24]. In addition, Stemberger et al. [28] found that the transfer of very high-quality antigen-specific $\mathrm{T}$ cells, which is additionally determined by the subset differentiation, can provide exceptionally robust protection against pathogens even at very low cell numbers. Together, these data suggest that the application of the TCR-pMHC $k_{\text {off }}$-rate assay for the identification of highly functional $\mathrm{T}$ cell populations could lead to a reduction of the number of $\mathrm{T}$ cells needed for the adoptive transfer, reducing costs and possible side effects.

\section{Clinical relevance}

The selection of $\mathrm{T}$ cell receptors most suitable for genetic engineering of autologous $\mathrm{T}$ cell populations could be guided by the $k_{\text {off }}$-rate assay, a technology which has the capacity to broaden the applicability of adoptive $\mathrm{T}$ cell therapy. Putatively, individual high-avidity $\mathrm{T}$ cells could be identified in a high-throughput manner via the $k_{\text {off }}$-rate assay, 
$a$ $b$

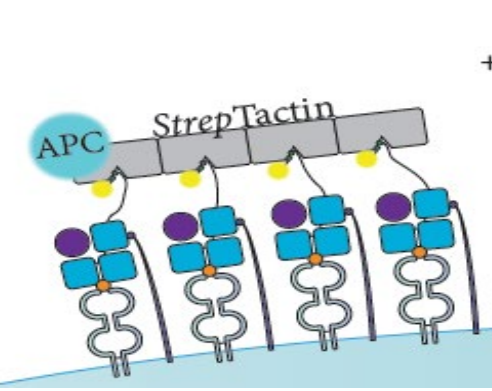

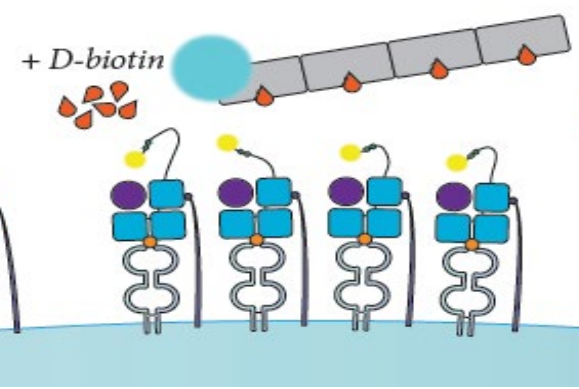

$C$

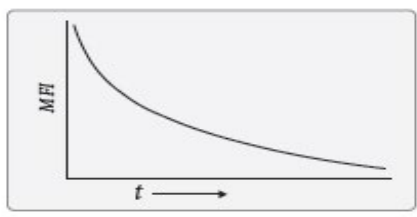

Figure 2: $\boldsymbol{k}_{\text {off }}$-rate assay

a. Stable, antigen-specific identification of cells by the koff-rate assay also relies on the cooperative binding of several pMHCs to TCRs of interest. MHCs are noncovalently multimerised to fluorescent StrepTactin via C-terminal StrepTag sequences, which terminate in a cysteine residue for fluorophore conjugation.

b. Addition of dissolved D-biotin causes displacement of the StrepTag sequences, separation, and diffusion of the Tactin multimer, detectable by a rapid loss of fluorescent intensity. Consequently, truly monomeric pMHC bound remains bound to the T cell surface and TCRs of interest.

c. Decay in fluorescent intensity over the ensuing seconds to minutes corresponds to the stochastic, monomeric dissociation and diffusion of pMHC molecules from their cognate TCRs, demonstrating in real-time the koff -rate of the TCR-pMHC interaction in question.

with TCR extraction and subsequent recombinant TCR expression $[24,29,30]$. This process has the capacity to permit the identification of ideal, high-avidity, pathogen-specific TCRs, with opportunities for the characterisation of TCRs bearing the greatest clinical relevance, and the development of novel cellular therapeutics. In patients who have successfully cleared or controlled infections, the $k_{\text {off }}$-rate assay has the potential to identify highly protective populations that have undergone clonal selection and evolution, enabling the directed extraction of TCRs for investigation and clinical application. Several investigators have demonstrated that the transfer of TCRs, in addition to the transfer of antigen specificity $[31,32]$, endows the recipient cells with functional avidities comparable to that of the original population $[30,33]$. Thus, the development of novel, $k_{\text {off }}$-rate-identified cellular therapeutics for infectious diseases is a tangible application of this technology to the clinical setting.

For example, Appay et al. [9] identify that $\mathrm{CD}^{+}{ }^{+}$-specific responses are crucial in the immune control of HIV, and that high avidity $\mathrm{T}$ cells result in superior control of viral replication by exerting a greater selective pressure on variable viruses, which as a result exhibit reduced replicative fitness, elegantly demonstrated in the work of VarelaRohena et al. [34] Similarly, Sud et al. [35] identify the importance of cytotoxic T cells in controlling infections of M. tuberculosis, in cooperation with other members of the adaptive immune system. Aside from the application of the $k_{\text {off }}$-rate assay to adoptive cell therapies, the assay has the potential to be of great advantage in diagnostic settings. Conceivably, the $k_{\text {off }}$-rate of antigen-specific T cell populations could demonstrate the quality of existing immunity and permit a degree of prognostication about response to illness to occur. Indeed, the work of Hadrup et al. [36] and Wikby et al. [37] have identified several features of an individual's immune system that are predictive of premature mortality in the aged. Given the short timeframe in which a $k_{o f f}$-rate assay can be performed, and the lack of unique equipment required to perform the assay, it would be easily integrated into the clinical environment. Furthermore, the quality of induced CD8 ${ }^{+} \mathrm{T}$ cell immune responses after vaccination are of critical importance and are able to be analysed with this assay. Identifying so-called non-responders early in vaccine development and monitoring cohort responses throughout the developmental course of new vaccines are two key applications of this assay. In the context of HIV, it has been shown that the variability of existing assays and the resultant inability to confidently correlate structural TCR-pMHC data with functional data has hampered the development of effective vaccines [9]. Additionally, Parida et al. [4] exemplify the importance of the development of both prophylactic and therapeutic vaccines for $M$. tuberculosis, as they lament that even with the availability and use of new anti-tuberculous medications, $M$. tuberculosis will continue to develop therapeutic resistance. Foreseeably, the $k_{\text {off }}$-rate assay could overcome practical difficulties by permitting the inference of functionality from structural avidity with regard to vaccine-induced $\mathrm{T}$ cell responses in both the pre-clinical and clinical phases of vaccine design and testing.

\section{Relevance to research}

The applications of the $k_{\text {off }}$-rate assay in basic research and clinics are manifold. Comparing $k_{\text {off }}$-rates of T cell populations after primary and secondary infection with Listeria monocytogenes, we found an unexpectedly large variance in the $k_{\text {off }}$-rates of $\mathrm{T}$ cells induced after primary infection, with a clear focussing to higher $k_{\text {off }}$-rates for some epitope specificies after secondary infection (unpublished data). This observation is in line with previous findings that narrowing of the repertoire towards higher avidity $\mathrm{T}$ cells [38] is largely due to failed recruitment of $\mathrm{T}$ cells with low structural avidity into secondary response [39].

The $k_{o f f}$-rate assay has also permitted the detailed investigation of chronic immune responses, further demonstrating its application to basic infection research. Infections in which sterilising immunity isn't achieved, including the pathogens HIV, CMV, EBV, and M. tuberculosis, are characterised by several mechanisms to control pathogen replication, including memory inflation [40]. A well studied example is the course of CMV infection during ageing [36,37], the studies of which have lead to the theory of 'The Clonal Changing of the Guard' [41]. According to this theory, high avidity $\mathrm{T}$ cells become exhausted and eventually deleted during ongoing infection. Due to reduced thymic output of novel, potentially high-avidity $\mathrm{T}$ cells, they are gradually replaced by sub-dominant clones [42], each of which has a lower affinity 
for MHC-presented viral antigens. This requires larger populations to prevent fulminant viral infection, such that global functional avidity is maintained, and so gradually occupy a larger proportion of the peripheral T cell pool. Pertinently, in a mouse model of chronic murine CMV infection and in accordance with this theory, we found a clear tendency towards inflating populations with decreasing $k_{\text {off }}$-rate during chronic infection (unpublished data). Further investigation of immune phenomena using the $k_{\text {off }}$-rate assay will indubitably yield new insights into the physiology of the adaptive immune system.

In summary, the TCR-pMHC $k_{\text {off }}$-rate assay allows for the accurate determination of the TCR-ligand avidity of cytotoxic T cells. This can be used for the identification and isolation of highly functional $\mathrm{T}$ cells to improve adoptive $\mathrm{T}$ cell therapy of severe infections, to monitor the quality of existing and induced immunity, and to gain insights into the course of infections in basic research.

\section{References}

1. World Health Organisation (2014) YLL By Cause, Age, and Sex 2000-2012. Geneva, Switzerland.

2. HIVIAIDS Programme, World Health Organisation (2012) WHO HIV Drug Resistance Report 2012. Geneva, Switzerland.

3. Global TB Programme, World Health Organisation. (2015) Global Tuberculosis Report 2015. Geneva, Switzerland.

4. Parida SK, Axelsson-Robertson R, Rao MV, Singh N, Master I, et al. (2015) Totally drug-resistant tuberculosis and adjunct therapies. J Intern Med 277: 388-405.

5. Schub A, Schuster IG, Hammerschmidt W, Moosmann A (2009) CMV-specific TCR-transgenic T cells for immunotherapy. J Immunol 183: 6819-6830.

6. Calmy A, Hirschel B, Cooper DA, Carr A (2009) A new era of antiretroviral drug toxicity. Antivir Ther 14: 165-179.

7. Goodrich JM, Mori M, Gleaves CA, Du Mond C, Cays M, et al. (1991) Early treatment with ganciclovir to prevent cytomegalovirus disease after allogeneic bone marrow transplantation. N Engl J Med 325: 1601-1607.

8. Saukkonen JJ, Cohn DL, Jasmer RM, Schenker S, Jereb JA, et al. (2006) An official ATS statement: hepatotoxicity of antituberculosis therapy. Am J Respir Crit Care Med 174: 935-952.

9. Appay V, Douek DC, Price DA (2008) CD8+ T cell efficacy in vaccination and disease. Nat Med 14: 623-628.

10. Stone JD, Chervin AS, Kranz DM (2009) T-cell receptor binding affinities and kinetics: impact on T-cell activity and specificity. Immunology 126: 165-176.

11. Stone JD, Kranz DM (2013) Role of T cell receptor affinity in the efficacy and specificity of adoptive T cell therapies. Front Immunol 4: 244

12. Zehn D, Lee SY, Bevan MJ (2009) Complete but curtailed T-cell response to very low-affinity antigen. Nature 458: 211-214.

13. Kalergis AM, Boucheron N, Doucey MA, Palmieri E, Goyarts EC, et al. (2001) Efficient $T$ cell activation requires an optimal dwell-time of interaction between the TCR and the pMHC complex. Nat Immunol 2: 229-234.

14. Derby M, Alexander-Miller M, Tse R, Berzofsky J (2001) High-avidity CTL exploit two complementary mechanisms to provide better protection against viral infection than low-avidity CTL. J Immunol. 166: 1690-1697.

15. Dutoit V, Rubio-Godoy V, Dietrich PY, Quiqueres AL, Schnuriger V, et al. (2001) Heterogeneous T-cell response to MAGE-A10(254-262): high avidity-specific cytolytic T lymphocytes show superior antitumor activity. Cancer Res 61: 58505856.

16. Zeh HJ, Perry-Lalley D, Dudley ME, Rosenberg SA, Yang JC (1999) High avidity CTLs for two self-antigens demonstrate superior in vitro and in vivo antitumor efficacy. J Immunol. 162. 989-994.

17. Nauerth M, Weissbrich B, Busch DH (2013) The clinical potential for koff-rate measurement in adoptive immunotherapy. Expert Rev Clin Immunol 9: 1151-1153.

18. Liedberg B, Nylander C, Lunström I (1983) Surface plasmon resonance for gas detection and biosensing. Sensors and Actuators 4: 299-304.
19. Irving M, Zoete V, Hebeisen M, Schmid D, Baumgartner P, et al. (2012) Interplay between $T$ cell receptor binding kinetics and the level of cognate peptide presented by major histocompatibility complexes governs CD8+ T cell responsiveness. J Biol Chem 287: 23068-78.

20. Wang XL, Altman JD (2003) Caveats in the design of MHC class I tetramer/ antigen-specific $\mathrm{T}$ lymphocytes dissociation assays. J Immunol Methods 280: 25-35.

21. Huang J, Zarnitsyna VI, Liu B, Edwards LJ, Jiang N, et al. (2010) The kinetics of two-dimensional TCR and pMHC interactions determine T-cell responsiveness. Nature 464: 932-936.

22. Huppa JB, Axmann M, Mörtelmaier MA, Lillemeier BF, Newell EW, et al. (2010) TCR-peptide-MHC interactions in situ show accelerated kinetics and increased affinity. Nature 463: 963-967.

23. Knabel M, Franz TJ, Schiemann M, Wulf A, Villmow B, et al. (2002) Reversible $\mathrm{MHC}$ multimer staining for functional isolation of T-cell populations and effective adoptive transfer. Nat Med 8: 631-637.

24. Nauerth M, Weißbrich B, Knall R, Franz T, Dössinger G, et al. (2013) TCR ligand koff rate correlates with the protective capacity of antigen-specific CD8+ T cells for adoptive transfer. Sci Transl Med 5: 192ra87.

25. Hebeisen M, Allard M, Gannon PO, Schmidt J, Speiser DE, et al. (2015) Identifying Individual T Cell Receptors of Optimal Avidity for Tumor Antigens. Front Immunol 6: 582

26. Gannon PO, Wieckowski S, Baumgaertner P, Hebeisen M, Allard M, et al (2015) Quantitative TCR:pMHC Dissociation Rate Assessment by NTAmers Reveals Antimelanoma T Cell Repertoires Enriched for High Functional Competence. J Immunol 195: 356-366.

27. Weissbrich B, Nauerth M, Busch DH (2013) Adoptive immunotherapy: New assay for the identification of $T$ cells with optimal avidity. Oncoimmunology 2 e26199.

28. Stemberger C, Graef P, Odendahl M, Albrecht J, Dössinger G, et al. (2014 Lowest numbers of primary $\mathrm{CD} 8(+) \mathrm{T}$ cells can reconstitute protective immunity upon adoptive immunotherapy. Blood 124: 628-637.

29. Dössinger G, Bunse M, Bet J, Albrecht J, Paszkiewicz PJ, et al. (2013) MHC Multimer-Guided and Cell Culture-Independent Isolation of Functional T Cell Receptors from Single Cells Facilitates TCR Identification for Immunotherapy PLoS ONE 8: e61384.

30. Wilde S, Sommermeyer D, Frankenberger B, Schiemann M, Milosevic S, et al (2009) Dendritic cells pulsed with RNA encoding allogeneic MHC and antigen induce $T$ cells with superior antitumor activity and higher TCR functional avidity. Blood 114: 2131-2139.

31. Clay TM, Custer MC, Sachs J, Hwu P, Rosenberg SA, et al. (1999) Efficient transfer of a tumor antigen-reactive TCR to human peripheral blood lymphocytes confers anti-tumor reactivity. J Immunol. 163: 507-513.

32. Morgan RA, Dudley ME, Yu YY, Zheng Z, Robbins PF, et al. (2003) High efficiency TCR gene transfer into primary human lymphocytes affords avid recognition of melanoma tumor antigen glycoprotein 100 and does not alter the recognition of autologous melanoma antigens. J Immunol 171: 3287-3295.

33. Johnson LA, Heemskerk B, Powell DJ, Cohen CJ, Morgan RA, et al. (2006) Gene transfer of tumor-reactive TCR confers both high avidity and tumor reactivity to nonreactive peripheral blood mononuclear cells and tumorinfiltrating lymphocytes. J Immunol. 177: 6548-6559.

34. Varela-Rohena A, Molloy PE, Dunn SM, Li Y, Suhoski MM, et al. (2008) Contro of HIV-1 immune escape by CD8 T cells expressing enhanced T-cell receptor. Nat Med 14: 1390-1395

35. Sud D, Bigbee C, Flynn JL, Kirschner DE (2006) Contribution of CD8+ T cells to control of Mycobacterium tuberculosis infection. J Immunol 176: 4296-4314.

36. Hadrup SR, Strindhall J, Kollgaard T, Seremet T, Johansson B, et al. (2006) Longitudinal studies of clonally expanded CD8 T cells reveal a repertoire shrinkage predicting mortality and an increased number of dysfunctional cytomegalovirus-specific T cells in the very elderly. J Immunol. 176: 2645-2653.

37. Wikby A, Johansson B, Olsson J, Lofgren S, Nilsson BO, et al. (2002) Expansions of peripheral blood CD8 T-lymphocyte subpopulations and an association with cytomegalovirus seropositivity in the elderly: the Swedish NONA immune study. Exp Gerontol 37: 445-453. 
Citation: Nauerth M, Wing K, Korner H, Busch D (2016) Relevance of the T cell Receptor-Ligand Avidity for Immunity to Infection. J Microb Biochem Technol 8: 131-135. doi: 10.4172/1948-5948.1000275

38. Busch DH, Pamer EG (1999) T cell affinity maturation by selective expansion during infection. J Exp Med 189: 701-710.

39. Savage PA, Boniface JJ, Davis MM (1999) A kinetic basis for T cell receptor repertoire selection during an immune response. Immunity 10: 485-492.

40. Snyder CM, Cho KS, Bonnett EL, van Dommelen S, Shellam GR, et al. (2008) Memory inflation during chronic viral infection is maintained by continuous production of short-lived, functional T cells. Immunity 29: 650-659.
41. Buchholz VR, Neuenhahn M, Busch DH (2011) CD8+ T cell differentiation in the aging immune system: until the last clone standing. Curr Opin Immunol 23: $549-554$

42. Holtappels R, Simon CO, Munks MW, Thomas D, Deegen $P$, et al. (2008) Subdominant CD8 T-cell epitopes account for protection against cytomegalovirus independent of immunodomination. J Virol 82: 5781-5796. 